\title{
High White Blood Cell Count Is a Risk Factor for Contrast-Induced Nephropathy following Mechanical Thrombectomy for Acute Ischemic Stroke
}

\author{
Yuki Yamamoto ${ }^{a}$ Nobuaki Yamamoto $^{a}$ Yasuhisa Kanematsu $^{b}$ \\ Kazutaka Kuroda $^{a} \quad$ Izumi Yamaguchi $^{b}$ Takeshi Miyamoto ${ }^{b}$ Shu Sogabe ${ }^{b}$ \\ Kenji Shimadab ${ }^{b}$ Yasushi Takagi $^{b}$ Yuishin Izumi ${ }^{a}$ \\ a Department of Neurology, Institute of Biomedical Sciences, Tokushima University, \\ Tokushima, Japan; ${ }^{b}$ Department of Neurosurgery, Institute of Biomedical Sciences, \\ Tokushima University, Tokushima, Japan
}

\section{Keywords}

Contrast-induced nephropathy · Mechanical thrombectomy · White blood cell count

\section{Abstract}

Background: Although mechanical thrombectomy is a standard endovascular therapy for patients with acute ischemic stroke (AIS), the incidence of and risk factors for contrast-induced nephropathy (CIN) following mechanical thrombectomy are infrequently reported. Objectives: The aim of this study was to investigate the incidence and risk factors for CIN following mechanical thrombectomy for AIS, and whether the incidence of CIN is related to a poor prognosis. Methods: We examined consecutive patients who underwent a mechanical thrombectomy in the period from January 2014 to March 2018. The patients' clinical backgrounds, treatments, and clinical prognoses were analyzed. CIN was defined as an increase in the serum creatinine level of $\geq 44.2 \mu \mathrm{mol} / \mathrm{L}(0.5 \mathrm{mg} / \mathrm{dL})$ or $25 \%$ above baseline within $72 \mathrm{~h}$ after exposure to the contrast medium. Results: In total, 80 patients ( 46 men and 34 women aged $74.5 \pm 11.5$ years) who met our inclusion criteria were analyzed. CIN occurred in $8.8 \%(7 / 80)$ of the patients following mechanical thrombectomy. Although no patients needed permanent dialysis, 1 required temporary dialysis. The median amount of contrast medium was $109 \mathrm{~mL}$. A comparison between the groups with and without CIN showed a significant difference in white blood cell (WBC) count at the time of admission $\left(11.6 \pm 2.7 \times 10^{3} / \mu \mathrm{L}\right.$ and $\left.8.1 \pm 2.7 \times 10^{3} / \mu \mathrm{L} ; p<0.01\right)$ and 
the cut-off value was $9.70 \times 10^{3} / \mu \mathrm{L}$. In multivariate analysis, contrast volume/estimated glomerular filtration rate by creatinine and WBC count were significantly associated with the incidence of $\mathrm{CIN}$, with odds ratios of $1.64(95 \% \mathrm{Cl} 1.02-2.65 ; p=0.04)$ and $1.61(95 \% \mathrm{Cl} 1.15-2.25$; $p<0.01$ ), respectively. Conclusions: This study found that CIN occurred in $8.8 \%$ of patients with AIS following mechanical thrombectomy. High WBC count was associated with an increased risk of $\mathrm{CIN}$ and may be helpful for predicting $\mathrm{CIN}$.

(C) 2020 The Author(s)

Published by S. Karger AG, Basel

\section{Introduction}

Contrast-induced nephropathy (CIN) is a serious complication of angiographic procedures resulting from the administration of iodinated contrast medium. The most commonly used definition of CIN is a rise in serum creatinine (Scr) level of $\geq 44.2 \mu \mathrm{mol} / \mathrm{L}(0.5 \mathrm{mg} / \mathrm{dL}$ ) or $25 \%$ above baseline within $72 \mathrm{~h}$ after exposure to the contrast medium [1]. It is generally a transient and reversible form of acute renal failure; however, some patients with CIN have a course that is severe enough to require dialysis. The development of CIN is also associated with a longer hospital stay and increased morbidity and mortality, in addition to higher costs [2]. Risk factors for CIN include advanced age, diabetes mellitus, preexisting renal failure, reduced renal perfusion from hypovolemia or congestive heart failure, and the use of a large amount of contrast medium or an ionic hyperosmolar contrast medium $[3,4]$.

Mechanical thrombectomy is a standard endovascular therapy for patients with acute ischemic stroke (AIS) [5]. The number of procedures being performed is increasing, but the incidence of and risk factors for CIN following mechanical thrombectomy are rarely reported. Here, we investigated the incidence and risk factors of CIN following mechanical thrombectomy.

\section{Materials and Methods}

\section{Population and Clinical Data}

We retrospectively examined 83 consecutive patients who underwent a mechanical thrombectomy for acute cerebral large-vessel occlusion (LVO) at our hospital between 1 January 2014 and 31 March 2018. After excluding 3 patients who had undergone maintenance dialysis at admission, 80 patients were analyzed.

$\mathrm{SCr}$ was measured at admission and for 2 consecutive days after the endovascular procedure. CIN was defined as an increase in SCr $\geq 44.2 \mu \mathrm{mol} / \mathrm{L}(0.5 \mathrm{mg} / \mathrm{dL})$ or $25 \%$ above baseline $72 \mathrm{~h}$ after exposure to the contrast medium. We compared clinical characteristics, laboratory data, and procedures between the groups with and without CIN.

The estimated glomerular filtration rate by creatinine (eGFRcreat) was calculated using the Japanese coefficient: $194 \times(\mathrm{SCr})^{-1.094} \times(\text { age in years })^{0.287} \times(0.739$ if female $)[6]$.

Vascular risk factors were recorded for each patient and defined as follows: (1) hypertension, a history of antihypertensive drug use, a systolic blood pressure $\geq 140 \mathrm{~mm} \mathrm{Hg}$ or diastolic blood pressure $\geq 90 \mathrm{~mm} \mathrm{Hg}$ at hospital discharge; (2) diabetes mellitus, current hypoglycemic drug use, a random glucose level $\geq 200 \mathrm{mg} / \mathrm{dL}$, or a glycosylated hemoglobin level $\geq 6.5 \%$ at admission; and (3) hyperlipidemia, a history of antihyperlipidemic drug use, and a serum low-density lipoprotein cholesterol level $\geq 140 \mathrm{mg} / \mathrm{dL}$. 
Table 1. Baseline characteristics of the participants

\begin{tabular}{|c|c|c|c|}
\hline & $\begin{array}{l}\text { CIN } \\
(n=7)\end{array}$ & $\begin{array}{l}\text { No CIN } \\
(n=73)\end{array}$ & $p$ value \\
\hline Age, years & $73.4 \pm 11.5$ & $74.6 \pm 11.6$ & 0.79 \\
\hline Male sex & $7(100)$ & $39(53.4)$ & $0.019 *$ \\
\hline Past ischemic stroke & $4(57.1)$ & 16 (21.9) & 0.06 \\
\hline Hypertension & $5(71.4)$ & $38(52.1)$ & 0.44 \\
\hline Diabetes mellitus & $2(28.6)$ & $16(21.9)$ & 0.65 \\
\hline Dyslipidemia & $2(28.6)$ & $11(15.1)$ & 0.32 \\
\hline Current smoking & $4(57.1)$ & $28(38.4)$ & 0.43 \\
\hline WBC count, $10^{3} / \mu \mathrm{L}$ & $11.6 \pm 2.7$ & $8.1 \pm 2.7$ & $<0.01^{*}$ \\
\hline Platelet count, $10^{3} / \mu \mathrm{L}$ & $258.9 \pm 121.2$ & $210.8 \pm 83.9$ & 0.17 \\
\hline Hemoglobin, g/dL & $12.3 \pm 1.8$ & $12.8 \pm 1.7$ & 0.47 \\
\hline LDL-C, mg/dL & $120.0 \pm 69.9$ & $102.0 \pm 33.1$ & 0.23 \\
\hline $\mathrm{TG}, \mathrm{mg} / \mathrm{dL}$ & $78.7 \pm 16.5$ & $119.7 \pm 104.7$ & 0.31 \\
\hline $\mathrm{CRP}, \mathrm{mg} / \mathrm{dL}$ & $1.2 \pm 2.4$ & $1.0 \pm 1.9$ & 0.84 \\
\hline Blood sugar, mg/dL & $158.1 \pm 68.6$ & $140 \pm 56.9$ & 0.45 \\
\hline $\mathrm{HbA}_{1 \mathrm{c}}, \%$ & $6.1 \pm 0.6$ & $6.1 \pm 1.1$ & 0.95 \\
\hline BUN, mg/dL & $21.3 \pm 9.2$ & $18.5 \pm 7.1$ & 0.35 \\
\hline $\mathrm{SCr}, \mathrm{mg} / \mathrm{dL}$ & $1.2 \pm 0.8$ & $0.8 \pm 0.3$ & 0.24 \\
\hline eGFRcreat, $\mathrm{mL} / \mathrm{min} / 1.73 \mathrm{~m}^{2}$ & $60.7 \pm 30.1$ & $67.4 \pm 21.9$ & 0.46 \\
\hline Contrast volume, mL & $109(60-150)$ & $109(30-250)$ & 0.83 \\
\hline Contrast volume/eGFRcreat & $2.57 \pm 2.26$ & $1.81 \pm 1.25$ & 0.16 \\
\hline Low-osmolar contrast agent use & $1(14.3)$ & 21 (28.8) & 0.67 \\
\hline NIHSS score & $19.0 \pm 7.1$ & $17.3 \pm 8.4$ & 0.60 \\
\hline IV rt-PA & $4(57.1)$ & $39(53.4)$ & 1.00 \\
\hline Number of passes & $1.6 \pm 1.1$ & $2.2 \pm 1.4$ & 0.26 \\
\hline Puncture to reperfusion time, min & $60.6 \pm 29.9$ & $77.6 \pm 37.7$ & 0.25 \\
\hline TICI grade $\geq 2 \mathrm{~b}$ & 7 (100) & $53(72.6)$ & 0.18 \\
\hline Perioperative aspirin use & $5(71.4)$ & $24(32.9)$ & 0.09 \\
\hline Number of days in hospital & $24.7 \pm 11.2$ & $17.7 \pm 9.2$ & 0.06 \\
\hline An mRS score of $0-2$ at 90 days & $1(14.3)$ & $23(31.5)$ & 0.67 \\
\hline
\end{tabular}

Values are expressed as $n(\%)$ or mean \pm SD.

Diagnosis of LVO was performed using magnetic resonance imaging (MRI), and no contrast medium was used for the diagnosis except in 1 patient who was contraindicated for MRI due to a pacemaker insertion. Mechanical thrombectomy was performed either alone or with intravenous recombinant tissue plasminogen activator (IV rt-PA), depending on the adaption. According to our institutional protocol, patients eligible for IV rt-PA were administered $0.6 \mathrm{mg} / \mathrm{kg}$ within $4.5 \mathrm{~h}$ of onset [7]. A stent retriever or aspiration catheter, or both, were used for mechanical thrombectomy. After mechanical thrombectomy, results were assessed according to the Thrombolysis in Cerebral Infarction grading scale [8]. Functional outcome was evaluated using the modified Rankin Scale score at 90 days.

All patients received either the nonionic low-osmolar contrastagentiohexol (Omnipaque ${ }^{\circledR}$ 300; Daiichi-Sankyo, Tokyo, Japan), iopamidol (Iopamiron ${ }^{\circledR} 300$; Bayer, Leverkusen, Germany), or the nonionic iso-osmolar contrast agent iodixanol (Visipaque ${ }^{\circledR} 270$; Daiichi-Sankyo) during the procedure.

\section{Statistical Analyses}

Results are expressed as mean \pm standard deviation or median (range) for quantitative variables and $n(\%)$ for categorical variables. Comparisons were performed with an analysis 


\section{Cerebrovascular Diseases Extra}

Cerebrovasc Dis Extra 2020;10:59-65 DOI: $10.1159 / 000507918$

(C) 2020 The Author(s). Published by S. Karger AG, Basel www.karger.com/cee

Yamamoto et al.: Contrast-Induced Nephropathy following Mechanical Thrombectomy
Fig. 1. Receiver operating characteristics (ROC) curve to determine the cut-off value of WBC count to predict CIN following mechanical thrombectomy. AUC, area under the curve; CIN, contrast-induced nephropathy; Sens, sensitivity; Spec, specificity; WBC, white blood cell.

Table 2. Results of the multivariate analysis

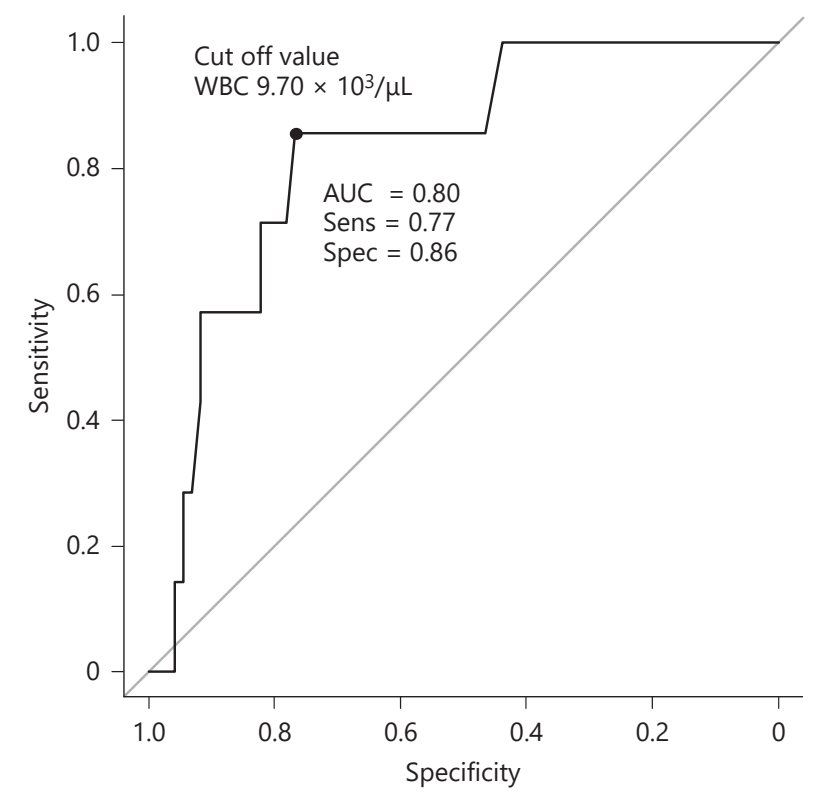

\begin{tabular}{lrrr}
\hline & OR & \multicolumn{1}{c}{$95 \%$ CI } & $p$ value \\
\hline Contrast volume/eGFRcreat & 1.64 & $1.02-2.65$ & $0.04^{*}$ \\
WBC count & 1.61 & $1.15-2.25$ & $<0.01^{*}$ \\
Age & 1.01 & $<0.01-0.83$ & 0.76 \\
Diabetes mellitus & 0.79 & $0.10-6.20$ & 0.82
\end{tabular}

of variance or the Mann-Whitney U test for continuous or ordinal variables, and the $\chi^{2}$ test for categorical variables was used in univariate analyses. All statistical tests were 2 -sided, and the significance $(p)$ level was set at 0.05 , unless stated otherwise. Perioperative variables as a known risk factor for CIN and appropriate factors with $p$ values $<0.05$ from the univariate analysis were used in the evaluation of the multivariate analysis. To calculate the sensitivity and specificity of parameters employed for biomarkers associated with CIN, we prepared a receiver-operating characteristics (ROC) curve. The results of multivariate analysis were expressed as adjusted odds ratios (ORs) and 95\% confidence intervals (CIs). All statistical analyses were performed using SPSS v20.0 for Windows (IBM, Armonk, NY, USA).

\section{Results}

We retrospectively examined 80 patients ( 46 men and 34 women aged $74.5 \pm 11.5$ years) who met our inclusion criteria. CIN occurred in 8.8\% (7/80) of the patients after mechanical thrombectomy. The baseline characteristics of the participants are summarized in Table 1. Although no patients needed permanent dialysis, 1 patient required temporary dialysis. A comparison between the groups with and without CIN showed a significant difference in sex ( 7 men and 0 women, respectively; $p=0.019$ ). In addition, white blood cell (WBC) count at admission was significantly higher in the CIN group than in the group without CIN $(11.6 \pm 2.7$ 
and $8.1 \pm 2.7 \times 10^{3} / \mu \mathrm{L}$, respectively; $p<0.01$ ). We used the ROC curve to determine the cut-off value of the WBC count (Fig. 1): $9.70 \times 10^{3} / \mu \mathrm{L}$. The ROC curve yielded an area under the curve of 0.80 (95\% CI 0.68-0.97), 77\% sensitivity, and $86 \%$ specificity. Multivariate analysis (Table 2) revealed that a high contrast volume/eGFRcreat rate and a high WBC count were significantly associated with the incidence of CIN, with an OR of 1.64 (95\% CI 1.02-2.65; $p=0.04$ ) and 1.61 (95\% CI 1.15-2.25; $p<0.01$ ), respectively. The development of CIN was not associated with a longer hospital stay or increased morbidity and mortality.

\section{Discussion}

This study shows that CIN following mechanical thrombectomy for AIS occurs at a rate of $8.8 \%$. This rate is similar to previous reports on neurointerventions $[9,10]$. CIN occurred in $7 / 80(8.8 \%)$ patients after carotid-artery stenting [9], and in 16/192 (8.3\%) patients after coil embolization with an aneurysmal subarachnoid hemorrhage [10]. Recently, CIN after mechanical thrombectomy occurred in $11 / 330(3.3 \%)$ patients [11], a lower incidence than in our study, probably due to the younger study population ( $63.9 \pm 15.8 \mathrm{vs.} 74.5 \pm 11.5$ years). In addition, CIN was defined in that study [11] as an increase in Scr $\geq 26.5 \mu \mathrm{mol} / \mathrm{L}$ or $1.5 \times$ baseline Scr. Irrespective of the route of administration, iodine contrast medium causes direct cytotoxicity to renal tubular cells, and ischemic injury by vasoconstriction of the renal peripheral circulation, both of which lead to CIN [12]. Therefore, the incidence of CIN is not highly dependent on the treatment procedure, but is rather influenced by contrast agents, individual patient factors, or other determinants. Since mechanical thrombectomy is essentially an urgent procedure, it may be limited to preventative options. In our study, patients with preoperative renal hypofunction had a high incidence of CIN. It is necessary to consider reducing the amount of contrast medium used in such cases.

The use of larger amounts of contrast medium is one of the classic risk factors for CIN [4]. In our study, the median amount of contrast medium was $109 \mathrm{~mL}$, less than the $236 \mathrm{~mL}$ used in the previous report [11]. Although our findings could not obtain a statistically significant difference in either contrast volume or procedure contents in the incidence of CIN, probably because of the small sample size, prolonging the procedure may involve increased use of contrast medium. Additionally, it should be noted that we used MRI to diagnose cerebral large-artery occlusion and did not use computed tomography angiography (CTA) and perfusion (CTP). CTP and its analysis software help quantitatively distinguish between the ischemic core and hypoperfused tissue [13]; thus, they are increasingly used to assist in AIS diagnosis and judgment of intervention decisions. However, Hopyan et al. [14] reported that when CTA and CTP were used to guide the emergency management of acute stroke, CIN occurred in 5/175 (2.9\%) patients, and the median use of contrast medium was $114 \mathrm{~mL}$. If mechanical thrombectomy is added after CTA and CTP examinations, the amount of contrast medium used is expected to exceed $200 \mathrm{~mL}$ and the incidence of CIN may be higher than that in our study.

In this study, we found that high preoperative WBC count was a novel risk factor (OR 1.61) for developing CIN following mechanical thrombectomy as a result of the multivariate analysis. A similar report showed an increase in inflammation-related molecules, including WBC count (especially neutrophils) in CIN following PCI [15]. The pathognomonic mechanism of CIN is not completely understood, but inflammation may play an important role in the initiation and extension phases of CIN [15]. In acute kidney injury, inflammatory cells infiltrate the interstitium of the kidney via cytokines and chemokines produced by both renal endothelial cells and proximal tubular epithelial cells. Inflammatory cells cause the release of oxygen radicals, vasoconstrictors, leukotrienes, and thromboxanes, leading to kidney damage

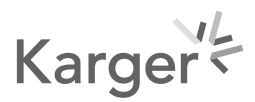


[16]. If the preoperative WBC count is high for various reasons, increased systemic inflammatory responses may promote the progression of CIN pathology.

The limitations of this study are that it was performed in a single center and involved a retrospective design. Further investigations are needed with a larger sample size to validate our results. Because only MRI was used for the diagnosis of LVO in our hospital, the incidence of CIN may have been lower than in the facility using contrast CT for diagnosis. Although we investigated the total WBC count, we could not evaluate their typing because of a lack of data that could not be examined at night. In future research, investigating WBC typing and other inflammation-related molecules may help predict CIN development.

We found that CIN occurred in 8.8\% (7/80) of AIS patients following mechanical thrombectomy. High WBC count was associated with an increased risk of CIN and might be helpful in predicting CIN. The development of CIN was not associated with a longer hospital stay or increased morbidity and mortality.

\section{Statement of Ethics}

The study protocol was approved by the research ethics committee of Tokushima University Hospital and consent was obtained from all participants.

\section{Disclosure Statement}

The authors have no conflicts of interest to declare.

\section{Funding Sources}

This research received no specific grant from any funding agency in the public, commercial, or not-for-profit sectors.

\section{Author Contributions}

Y.Y. designed the project, analyzed the data, and drafted the manuscript. N.Y. revised the manuscript for intellectual content and oversaw the study. Y.K., K.K., I.Y., T.M., S.S., and K.S. performed the treatment procedure and interpreted the data. Y.T. and Y.I. supervised this study.

\section{References}

1 Morcos SK, Thomsen HS, Webb JA. Contrast-media-induced nephrotoxicity: a consensus report. Contrast Media Safety Committee, European Society of Urogenital Radiology (ESUR). Eur Radiol. 1999;9(8):1602-13.

2 McCullough PA, Wolyn R, Rocher LL, Levin RN, O'Neill WW. Acute renal failure after coronary intervention: incidence, risk factors, and relationship to mortality. Am J Med. 1997 Nov;103(5):368-75.

3 Mehran R, Aymong ED, Nikolsky E, Lasic Z, Iakovou I, Fahy M, et al. A simple risk score for prediction of contrast-induced nephropathy after percutaneous coronary intervention: development and initial validation. J Am Coll Cardiol. 2004 Oct;44(7):1393-9.

4 Toprak 0. Conflicting and new risk factors for contrast induced nephropathy.J Urol. 2007 Dec;178(6):2277-83.

5 Goyal M, Menon BK, van Zwam WH, Dippel DW, Mitchell PJ, Demchuk AM, et al.; HERMES collaborators. Endovascular thrombectomy after large-vessel ischaemic stroke: a meta-analysis of individual patient data from five randomised trials. Lancet. 2016 Apr;387(10029):1723-31.

\section{Karger's}


6 Matsuo S, Imai E, Horio M, Yasuda Y, Tomita K, Nitta K, et al.; Collaborators developing the Japanese equation for estimated GFR. Revised equations for estimated GFR from serum creatinine in Japan. Am J Kidney Dis. 2009 Jun;53(6):982-92.

7 Yamaguchi T, Mori E, Minematsu K, Nakagawara J, Hashi K, Saito I, et al.; Japan Alteplase Clinical Trial (J-ACT) Group. Alteplase at $0.6 \mathrm{mg} / \mathrm{kg}$ for acute ischemic stroke within 3 hours of onset: Japan Alteplase Clinical Trial (J-ACT). Stroke. 2006 Jul;37(7):1810-5.

8 Yoo AJ, Simonsen CZ, Prabhakaran S, Chaudhry ZA, Issa MA, Fugate JE, et al.; Cerebral Angiographic Revascularization Grading Collaborators. Refining angiographic biomarkers of revascularization: improving outcome prediction after intra-arterial therapy. Stroke. 2013 Sep;44(9):2509-12.

9 Kato T, Sakai H, Tsujimoto M, Nishimura Y. Prolonged carotid sinus reflex is a risk factor for contrast-induced nephropathy following carotid artery stenting. AJNR Am J Neuroradiol. 2011 Mar;32(3):441-5.

10 Lee HG, Kim WK, Yeon JY, Kim JS, Kim KH, Jeon P, et al. Contrast-Induced Acute Kidney Injury after Coil Embolization for Aneurysmal Subarachnoid Hemorrhage. Yonsei Med J. 2018 Jan;59(1):107-12.

11 Diprose WK, Sutherland LJ, Wang MT, Barber PA. Contrast-Associated Acute Kidney Injury in Endovascular Thrombectomy Patients with and without Baseline Renal Impairment. Stroke. 2019 Dec;50(12):3527-31.

12 McCullough PA, Choi JP, Feghali GA, Schussler JM, Stoler RM, Vallabahn RC, et al. Contrast-Induced Acute Kidney Injury. J Am Coll Cardiol. 2016 Sep;68(13):1465-73.

13 Wintermark M, Flanders AE, Velthuis B, Meuli R, van Leeuwen M, Goldsher D, et al. Perfusion-CT assessment of infarct core and penumbra: receiver operating characteristic curve analysis in 130 patients suspected of acute hemispheric stroke. Stroke. 2006 Apr;37(4):979-85.

14 Hopyan JJ, Gladstone DJ, Mallia G, Schiff J, Fox AJ, Symons SP, et al. Renal safety of CT angiography and perfusion imaging in the emergency evaluation of acute stroke. AJNR Am J Neuroradiol. 2008 Nov;29(10):1826-30.

15 Yuan Y, Qiu H, Hu X, Luo T, Gao X, Zhao X, et al. Predictive value of inflammatory factors on contrast-induced acute kidney injury in patients who underwent an emergency percutaneous coronary intervention. Clin Cardiol. 2017 Sep;40(9):719-25.

16 Akcay A, Nguyen Q, Edelstein CL. Mediators of inflammation in acute kidney injury. Mediators Inflamm. 2009; 2009:137072. 